\title{
O método Multitraço-Multimétodo na validação de instrumentos de medida em dieta infantil
}

\author{
The Multitrait-Multimethod for validation of measurement instruments in child diet
}

\author{
REBECA REGLY MARCHITTO ${ }^{a}$ \\ MARIA ELISA BARROS ${ }^{b}$ \\ REGINA SERRÃO LANZILLOTTI ${ }^{\mathrm{c}}$ \\ HAYDÉE SERRÃO LANZILLOTTI ${ }^{\mathrm{d}}$
}

\begin{abstract}
Resumo
Objetivo: Avaliar a validade convergente e discriminante de um questionário destinado a responsáveis pela alimentação da criança sobre a adoção do óleo vegetal in natura de acréscimo na dieta infantil como estratégia de aumento da densidade energética. Metodologia: Algoritmo Matriz Multitraço-Multimétodo (MTMM) tendo como entrada matrizes de correlação dos escores dos itens do questionário referentes à Escala Likert. Resultados: Estudo amostral transversal com 300 responsáveis pela alimentação de crianças menores de dois anos realizado nos ambulatórios de pediatria de uma operadora de planos de saúde (SJM) e da Policlínica Piquet Carneiro da Universidade do Estado do Rio de Janeiro (PPC). Os valores da matriz Multitraço-Monométodo foram considerados de significância aceitável para as duas Unidades de Saúde, mas ao agregálas, há uma perda na qualidade inferencial das correlações, explicada pela diferença da estrutura funcional das unidades. A matriz de correlação Multitraço-Multimétodo apresentou um grande número de correlações fracas entre diferentes traços (itens) medidos em PPC/UERJ e SJM (método), variando de $-0,20$ a 0,20 , o que corrobora a validade discriminante. As correlações na diagonal de validade convergente para a PPC/UERJ variaram de $-0,41$ a 0,36 até 0,79 , o que denota validade convergente. De forma análoga, em SJM, as correlações variaram de $-0,23$ a 0,90 , podendo-se inferir sobre o alcance desta validade. Conclusão: O instrumento proposto apresentou evidências de validade discriminante e convergente.
\end{abstract}

Palavras-chave: Óleo vegetal, Dieta infantil, Multitraço-Multimétodo.

\footnotetext{
a Universidade do Estado do Rio de Janeiro, Rio de Janeiro, Brasil; ORCID: org/0000-0002-2845-0957 E-mail: rebecamarchitto95@gmail.com

b Universidade do Estado do Rio de Janeiro, Rio de Janeiro, Brasil; ORCID: 0000-0002-5538-7658 E-mail: melisavilela@gmail.com

${ }^{\mathbf{c}}$ Universidade do Estado do Rio de Janeiro, Rio de Janeiro, Brasil; ORCID: 0000-0001-7789-6843 E-mail: reginalanzillotti@gmail.com

${ }^{d}$ Universidade do Estado do Rio de Janeiro, Rio de Janeiro, Brasil; ORCID: 0000-0002-7252-4415 E-mail: haydeelan@gmail.com
} 


\begin{abstract}
Objective: To evaluate the convergent and discriminant validity of a questionnaire for those responsible for feeding the child on the adoption of in natura vegetable oil in addition to the children's diet as a strategy for increasing energy density. Methodology: MultitraitMultimethod Matrix Algorithm (MTMM) having as input the correlation matrices of the scores of the questionnaire items referring to the Likert Scale. Results: The crosssectional study with 300 responsible for feeding children under two years of age carried out in the pediatric outpatient clinics of a health plan operator (SJM) and in Health Service of the Piquet Carneiro Polyclinic of the State University of Rio de Janeiro (PPC). The values of the Multitrait-Monomethod matrix were considered of acceptable significance for the two Health Services, but when aggregating them, there is a loss in the inferential quality of the correlations, explained by the difference in the functional structure of the services. The Multitrait-Multimethod correlation matrix showed a large number of weak correlations between different traits (items) measured in PPC/UERJ and SJM (method), ranging from -0.20 to 0.20 , which corroborates the discriminant validity. The correlations in the convergent validity diagonal for the PPC / UERJ ranged from -0.41 to 0.36 up to 0.79 , these results denote convergent validity. In a similar way, at SJM, the correlations ranged from -0.23 to 0.90 which can be inferred about the scope of this validity. Conclusion: the proposed instrument presented evidence of discriminant and convergent validity.
\end{abstract}

Keywords: Vegetable oil, Child diet, Multitrait-Multimethod.

MSC2020: 34B60, 92D30

\title{
1. Introdução
}

A Política Nacional de Atenção Integral à Saúde da Criança (PNAISNCA) apresenta como um de seus eixos a "Atenção à Saúde de Crianças com Deficiência ou em Situações Específicas e de Vulnerabilidade". Uma das estratégias da PNAISNCA é a articulação entre a mãe e a rede pública de saúde que deve ser feita com a alta responsável pela maternidade com o uso da Caderneta de Saúde da Criança e agendamento do " 5 " Dia de Saúde Integral" da mãe e bebê[1]. O Ministério da Saúde operacionaliza esta articulação com publicações dirigidas às mães e profissionais da saúde no sentido da proposta "Dez passos para uma alimentação saudável" [2], a qual se vincula ao Eixo Estratégico II do PNAISNCA: Aleitamento Materno e Alimentação Complementar Saudável em que há a recomendação de acréscimo de óleo vegetal "in natura" nas refeições das crianças para aumentar a densidade energética da dieta [1]. 
O acréscimo do óleo vegetal à dieta da criança tem o intuito de supri-la de ácidos graxos poliinsaturados de cadeia longa. Lactentes alimentados com leite humano recebem apreciáveis quantidades de ácido araquidônico (AA) e ácido docosahexaenóico (DHA), que participam das reações bioquímicas das funções cognitivas (sistema nervoso) e visuais $[3,4,5]$.

Ressalta-se que o acréscimo do óleo vegetal na dieta infantil deverá ser in natura e imune de peróxidos, não deve ser aquecido para evitar reações como hidrólise, oxidação e polimerização do óleo, produzindo numerosos compostos de degradação [6].

Dentre os óleos vegetais, o de soja é o mais acessível ao consumo devido ao seu preço de mercado e dada a sua composição em ácidos graxos: 15,2\% saturado, 23,3\% monoinsaturado e $60,0 \%$ poliinsaturado [7].

Frequentemente, no atendimento nutricional ambulatorial presencia-se muita perplexidade entre os responsáveis pela alimentação da criança quando se prescreve o "óleo vegetal de acréscimo" para obter ganho de peso. As principais preocupações verbalizadas pelos responsáveis são: aumento do colesterol, alteração do sabor da preparação alimentar com consequente rejeição e possível aparecimento de diarreia. Apesar da recomendação do Ministério da Saúde existe uma atitude de resistência dos responsáveis a adoção desta prática na dieta da criança e entende-se como importante conhecer o que pensam e se adotariam a recomendação como estratégia para aumentar a densidade energética da dieta.

O objetivo deste estudo foi avaliar a validade convergente e discriminante de um questionário destinado a responsáveis pela alimentação da criança sobre a adoção do óleo vegetal in natura de acréscimo na dieta infantil como estratégia de aumento da densidade energética.

\section{Metodologia}

Trata-se de estudo transversal realizado em dois ambulatórios de pediatria, sendo que um deles refere-se a uma operadora de planos de saúde (SJM) no município de São João de Meriti (RJ) e o outro na Policlínica Piquet Carneiro da Universidade do Estado do Rio de Janeiro (PPC/UERJ), no município do Rio de Janeiro (RJ), no período de março de 2017 a janeiro de 2019. 


\subsection{Elaboração do questionário}

O questionário (Quadro 1) foi construído com as assertivas (itens) criadas a partir da verbalização dos acompanhantes das crianças durante as consultas de Nutrição, quando da prescrição do óleo vegetal comestível "in natura" para aumentar a densidade energética da dieta. Este apresenta duas dimensões: "Benefícios do acréscimo de óleos vegetais in natura na dieta infantil" designado por "P" para sete itens e "Possíveis problemas relacionados ao acréscimo de óleos vegetais in natura na dieta infantil" por "N", quatro itens. A meta era verificar o construto "Adoção do óleo vegetal in natura de acréscimo na dieta infantil" [8]. Os itens foram respondidos segundo a escala Likert e receberam escores em ordem decrescente, ou seja, 5 (concordo muito); 4 (concordo); 3 (nem concordo /nem discordo); 2 (discordo) e 1 (discordo muito).

$\mathrm{Na}$ aplicação do questionário cada sentença foi lida e solicitado ao entrevistado que indicasse seu grau de concordância ou discordância com cada item. O entrevistado foi orientado a dar apenas uma resposta para cada item do questionário.

Quadro 1. Itens do questionário para avaliar a adoção do óleo vegetal in natura de acréscimo na dieta infantil. Rio de Janeiro, RJ, 2013.

\begin{tabular}{|c|c|}
\hline Itens & Descrição \\
\hline \multicolumn{2}{|c|}{$\begin{array}{l}\text { Fator 1: "Benefícios do acréscimo de óleos vegetais in natura na dieta infantil" } \\
\qquad(\mathrm{P}=\text { positivo) }\end{array}$} \\
\hline $\mathrm{i} 4-\mathrm{P}$ & $\begin{array}{l}\text { Colocar óleo vegetal cru na comida da criança melhora a situação da } \\
\text { criança com baixo peso. }\end{array}$ \\
\hline i6-P & $\begin{array}{l}\text { Usar óleo vegetal cru na comida da criança melhora a imunidade da } \\
\text { criança. }\end{array}$ \\
\hline i7-P & $\begin{array}{l}\text { Se eu acrescentar óleo vegetal cru na comida da criança, ela não ficará } \\
\text { obesa. }\end{array}$ \\
\hline i9-P & É bom para a criança colocar óleo vegetal cru na mamadeira \\
\hline i10-P & $\begin{array}{l}\text { Usar óleo vegetal cru na comida da criança melhora sua resistência a } \\
\text { doenças transmissíveis. }\end{array}$ \\
\hline i13-P & O acréscimo de óleo vegetal cru não eleva o colesterol da criança. \\
\hline
\end{tabular}


i15-P O certo é não refogar os alimentos da criança e colocar o óleo vegetal cru depois da papa salgada pronta.

Fator 2 "Possíveis problemas relacionados ao acréscimo de óleos vegetais in natura na dieta infantil" ( $\mathrm{N}=$ negativo).

\begin{tabular}{|c|l|}
\hline i1-N & $\begin{array}{l}\text { Colocar óleo vegetal cru na comida da criança piora a situação da } \\
\text { criança com baixo peso. }\end{array}$ \\
\hline i3-N & Colocar óleo vegetal cru na comida da criança causa náuseas. \\
\hline i8-N & Colocar óleo vegetal cru na comida da criança causa vômito. \\
\hline i14-N & O uso de óleo vegetal cru todos os dias provoca diarreia. \\
\hline
\end{tabular}

Fonte: $[8, p .471]$

\subsection{Grupo de interesse}

Participaram do estudo 300 mães e/ou acompanhantes de crianças, as quais aguardavam a consulta na sala de espera do ambulatório de Pediatria da Policlínica Piquet Carneiro/UERJ ( $n=150)$ e da operadora de planos de saúde (SJM) no município de São João de Meriti $(n=150)$.

Elegeu-se como critério de inclusão para a aplicação do questionário que os entrevistados fossem os responsáveis pela alimentação da criança, seja comprando alimentos, preparando-os ou servindo suas refeições, uma vez que esta situação pode ser considerada intervenção de caráter dietético e, ainda, que as crianças não ultrapassassem dois anos de idade, de acordo com o Guia Alimentar para crianças menores de 2 anos [2]. A coleta de dados em ambos locais foi realizada por uma acadêmica de Nutrição devidamente treinada, em horário de maior fluxo de atendimento.

\subsection{Procedimento metodológico}

A fim de avaliar a validade e confiabilidade do questionário foi utilizado o método da Matriz multitraço-multimétodo proposto por Campbell e Fiske [4]. Os autores apresentaram um procedimento inferencial matemático como um método para estabelecer a validade de construto de medidas psicológicas. Particularmente neste estudo, o comportamento de responsáveis pela alimentação da criança em relação a uma prática dietética recomendada pelo Ministério da Saúde. 
Esta metodologia envolve uma matriz de correlações dispostas de uma maneira que promova a avaliação da validade do construto, sendo que no presente estudo, "adoção do uso do óleo vegetal de acréscimo na dieta infantil". Validade refere-se à extensão para a qual uma medida psicológica de fato mede o conceito que se propõe medir. Esta abordagem requer que vários traços (T: atitudes, características de personalidade, comportamentos) sejam avaliados por vários métodos (M: testes psicológicos alternativos, modalidades alternativas de avaliação, ocasiões separadas de aplicação do teste). O resultado é uma matriz de correlação $\mathrm{T} \times \mathrm{M}$ que é interpretada em relação às validades convergente e discriminante do instrumento de mensuração proposto (Tabela1). Nesta matriz os traços são indicados pelas letras A, B e C e os métodos 1, 2 e 3 são constituídos por três traços. A Tabela 1 é a matriz de correlação $3 \times 3$ organizada de modo que as correlações entre os diferentes traços estejam aninhados dentro de cada método. O MTMM é uma matriz de correlação simétrica, tendo na diagonal as estimativas de confiabilidade calculadas pelo alfa de Cronbach referentes às medidas, que são inseridas e representam a consistência interna do inventário de comportamento.

Campbell e Fiske [4] observam que a diagonal de confiabilidade deve conter os maiores coeficientes da matriz; ou seja, a medida deve estar mais fortemente correlacionada consigo mesmo do que com qualquer outro indicador na matriz MTMM. Esta matriz consiste em dois tipos gerais de blocos de coeficientes: (1) blocos monométodo, que contêm correlações entre indicadores derivados do mesmo método ou ocasião de teste; e (2) blocos heterométodo, que contêm correlações entre indicadores avaliados por diferentes métodos ou ocasiões de teste. O interesse é a diagonal de validade que corresponde à diagonal dentro de cada bloco heterométodo. Correlações na diagonal de validade representam estimativas de validade convergente, ou seja, diferentes medidas de construtos teoricamente semelhantes devem ser fortemente interrelacionadas. $\mathrm{Na}$ matriz MTMM, a validade convergente é evidenciada por fortes correlações entre os métodos que medem o mesmo traço, isto é, coeficientes monotraço-heterométodo. As correlações fora da diagonal dos blocos heterométodo revelam validade discriminante, ou seja, medidas de construtos teoricamente distintos não devem ser altamente intercorrelacionados. A validade discriminante na matriz MTMM é evidenciada por correlações mais fracas entre diferentes traços medidos por diferentes métodos, ou seja, coeficientes heterotraço-heterométodo. Ressalta-se o uso da correlação ordinal de Spearman, tendo em vista que a mensuração comportamental utilizou a escala Likert. 
Tabela 1: Matriz simétrica de correlações Multitraço-Multimétodo - MTMM

\begin{tabular}{ccccccccccc}
\hline Métodos & Traços & A & B & C & A & B & C & A & B & C \\
\hline & A & 1,00 & & & & & & & & \\
Método 1 & B & $\mathrm{r}_{\mathrm{AB} 1}$ & 1,00 & & & & & & & \\
& $\mathrm{C}$ & $\mathrm{r}_{\mathrm{AC} 1}$ & $\mathrm{r}_{\mathrm{BC} 1}$ & 1,00 & & & & & &
\end{tabular}

$\begin{array}{llllll}\mathrm{A} & \mathrm{rAA} 2 & \mathrm{r}_{\mathrm{BA} 2} & \mathrm{r}_{\mathrm{CA} 2} & 1,00 & \\ \mathrm{~B} & \mathrm{r}_{\mathrm{AB} 2} & \mathrm{r}_{\mathrm{BB} 2} & \mathrm{r}_{\mathrm{CB} 2} & \mathrm{r}_{\mathrm{AB} 2} & 1,00\end{array}$

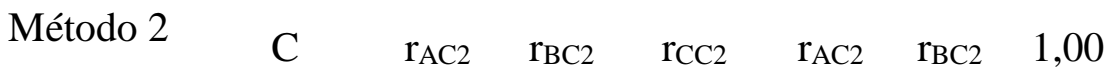

$\begin{array}{lllllllllll} & \mathrm{A} & \mathrm{r}_{\mathrm{AA} 3} & \mathrm{r}_{\mathrm{BA} 3} & \mathrm{r}_{\mathrm{CA} 3} & \mathrm{r}_{\mathrm{AA} 3} & \mathrm{r}_{\mathrm{BA} 3} & \mathrm{r}_{\mathrm{CA} 3} & 1,00 & & \\ & \mathrm{~B} & \mathrm{r}_{\mathrm{AB} 3} & \mathrm{r}_{\mathrm{BB} 3} & \mathrm{r}_{\mathrm{CB} 3} & \mathrm{r}_{\mathrm{AB} 3} & \mathrm{r}_{\mathrm{BB} 3} & \mathrm{r}_{\mathrm{CB} 3} & \mathrm{r}_{\mathrm{AB} 3} & 1,00 & \\ \text { Método 3 } & \mathrm{C} & \mathrm{r}_{\mathrm{AC} 3} & \mathrm{r}_{\mathrm{BC} 3} & \mathrm{r}_{\mathrm{CC} 3} & \mathrm{r}_{\mathrm{AC} 3} & \mathrm{r}_{\mathrm{BC} 3} & \mathrm{r}_{\mathrm{CC} 3} & \mathrm{r}_{\mathrm{AC} 3} & \mathrm{r}_{\mathrm{BC} 3} & 1,00\end{array}$

Nota: rABi, rAci, rAAi: correlações, i=1,2,3.

Fonte: [4] (adaptado).

A pesquisa foi aprovada pelo Comitê de Ética em Pesquisa da Universidade do Estado do Rio de Janeiro (RJ), sob o protocolo número 006.3.2008. Os participantes assinaram o Termo de Consentimento Livre e Esclarecido.

\section{Resultados}

A tabela 2 apresenta a matriz MTMM obtida após a aplicação do questionário nos dois ambulatórios de pediatria das unidades de saúde. 
Tabela 2: Diagramas das matrizes Multitraço-Monométodo (A e B ) e MultitraçoMultimétodo C (A vs B)

\begin{tabular}{|c|c|c|c|c|c|c|c|c|c|c|c|}
\hline \multicolumn{12}{|c|}{ Matriz multitraço-monométodo: Ambulatórios da Policlínica Piquet Carneiro (PPC) (A) n=150 } \\
\hline & i4Px & i6Px & i7Px & i9Px & $\mathrm{i} 10 \mathrm{Px}$ & i13Px & i15Px & $\mathrm{i} 1 \mathrm{Nx}$ & $\mathrm{i} 3 \mathrm{Nx}$ & i8Nx & $\mathrm{i} 14 \mathrm{Nx}$ \\
\hline i4Px & 1,00 & & & & & & & & & & \\
\hline i6Px & 0,67 & 1,00 & & & & & & & & & \\
\hline i7Px & 0,63 & 0,61 & 1,00 & & & & & & & & \\
\hline i9Px & 0,42 & 0,30 & $\mathbf{0 , 3 7}$ & 1,00 & & & & & & & \\
\hline i10Px & 0,59 & 0,63 & 0,58 & 0,43 & 1,00 & & & & & & \\
\hline i13Px & 0,55 & 0,60 & 0,49 & $0,21^{*}$ & 0,43 & 1,00 & & & & & \\
\hline i15Px & 0,47 & 0,35 & 0,38 & 0,32 & 0,42 & $\mathbf{0 , 3 8}$ & 1,00 & & & & \\
\hline $\mathrm{i} 1 \mathrm{Nx}$ & $-0,35$ & $-0,26$ & $-0,29$ & $-0,20$ & $-0,31$ & $-0,33$ & $-0,41$ & 1,00 & & & \\
\hline $\mathrm{i} 3 \mathrm{Nx}$ & $-0,3$ & $-0,34$ & $-0,26$ & $-0,18$ & $-0,32$ & $-0,31$ & $-0,33$ & 0,38 & 1,00 & & \\
\hline $\mathrm{i} 8 \mathrm{Nx}$ & $-0,29$ & $-0,27$ & $-0,23$ & $-0,24$ & $-0,26$ & $-0,28$ & $-0,40$ & 0,49 & 0,79 & 1,00 & \\
\hline $\mathrm{i} 14 \mathrm{Nx}$ & $-0,30$ & $-0,34$ & $-0,31$ & $-0,15^{*}$ & $-0,35$ & $-0,35$ & $-0,34$ & 0,49 & 0,70 & 0,72 & 1,00 \\
\hline \multicolumn{12}{|c|}{ Matriz multitraço-monométodo: Ambulatórios de São João de Meriti (SJM) (B) n=150 } \\
\hline & i4Py & i6Рy & i7Py & i9Py & i10Py & i13Py & i15Py & $\mathrm{i} 1 \mathrm{Ny}$ & i3Ny & i8Ny & i14Ny \\
\hline i4Py & 1,00 & & & & & & & & & & \\
\hline i6Py & 0,71 & 1,00 & & & & & & & & & \\
\hline i7Py & 0,54 & $\mathbf{0 , 4 1}$ & 1,00 & & & & & & & & \\
\hline i9Py & 0,43 & 0,41 & 0,43 & 1,00 & & & & & & & \\
\hline i10Py & 0,56 & 0,59 & 0,41 & 0,40 & 1,00 & & & & & & \\
\hline i13Py & 0,36 & 0,33 & 0,33 & 0,42 & $\mathbf{0 , 3 7}$ & 1,00 & & & & & \\
\hline i15Py & 0,54 & 0,48 & 0,53 & 0,57 & 0,51 & $\mathbf{0 , 5 3}$ & 1,00 & & & & \\
\hline i1Ny & $-0,25$ & $-0,28$ & $-0,18$ & $-0,07$ & $-0,24$ & $-0,14$ & $-0,23$ & 1,00 & & & \\
\hline i3Ny & $-0,16$ & $-0,26$ & $-0,21$ & $-0,10^{*}$ & $-0,26$ & $-0,16$ & $-0,30$ & 0,62 & 1,00 & & \\
\hline i8Ny & $-0,17$ & $-0,26$ & $-0,20$ & $-0,14 *$ & $-0,28$ & $-0,19$ & $-0,27$ & 0,64 & $\mathbf{0 , 8 8}$ & 1,00 & \\
\hline $\mathrm{i} 14 \mathrm{Ny}$ & $-0,13$ & $-0,17$ & $-0,18$ & $-0,14^{*}$ & $-0,23$ & $-0,18$ & $-0,31$ & 0,61 & 0,82 & 0,9 & 1,00 \\
\hline \multicolumn{12}{|c|}{ Matriz multitraço-multimétodo $C$ (A vs B) $n=150$} \\
\hline & i4Px & i6Px & i7Px & i9Px & i10Px & i13Px & i15Px & $\mathrm{i} 1 \mathrm{Nx}$ & $\mathrm{i} 3 \mathrm{Nx}$ & $\mathrm{i} 8 \mathrm{Nx}$ & $\mathrm{i} 14 \mathrm{Nx}$ \\
\hline i4Px & 1,00 & & & & & & & & & & \\
\hline i6Px & 0,71 & 1,00 & & & & & & & & & \\
\hline i7Px & 0,54 & $\mathbf{0 , 4 1}$ & 1,00 & & & & & & & & \\
\hline i9Px & 0,43 & 0,40 & 0,43 & 1,00 & & & & & & & \\
\hline i10Px & 0,56 & 0,59 & 0,41 & 0,40 & 1,00 & & & & & & \\
\hline i13Px & 0,36 & 0,33 & 0,33 & 0,42 & 0,37 & 1,00 & & & & & \\
\hline i15Px & 0,54 & 0,48 & 0,53 & 0,57 & 0,51 & $\mathbf{0 , 5 3}$ & 1,00 & & & & \\
\hline $\mathrm{i} 1 \mathrm{Nx}$ & $-0,25$ & $-0,28$ & $-0,18$ & $-0,07 *$ & $-0,24$ & $-0,14$ & $-0,23$ & 1,00 & & & \\
\hline $\mathrm{i} 3 \mathrm{Nx}$ & $-0,16$ & $-0,26$ & $-0,21$ & $-0,10^{*}$ & $-0,26$ & $-0,16$ & $-0,3$ & 0,62 & 1,00 & & \\
\hline $\mathrm{i} 8 \mathrm{Nx}$ & $-0,17$ & $-0,26$ & $-0,20$ & $-0,14$ & $-0,28$ & $-0,19$ & $-0,27$ & 0,64 & 0,89 & 1,00 & \\
\hline \multirow[t]{2}{*}{$\mathrm{i} 14 \mathrm{Nx}$} & $-0,13$ & $-0,17$ & $-0,18$ & $-0,14$ & $-0,23$ & $-0,18$ & $-0,31$ & 0,61 & 0,82 & 0,90 & 1,00 \\
\hline & $\mathrm{i} 4 \mathrm{Px}$ & i6Px & i7Px & i9Px & i10Px & i13Px & i15Px & i1Nx & $\mathrm{i} 3 \mathrm{Nx}$ & i8Nx & $\mathrm{i} 14 \mathrm{Nx}$ \\
\hline i4Py & 0,12 & 0,10 & $-0,06 *$ & $0,00^{*}$ & $-0,04 *$ & $0,12^{*}$ & 0,04 & $0,05^{*}$ & $-0,02 *$ & $0,00^{*}$ & $-0,02 *$ \\
\hline
\end{tabular}




\begin{tabular}{|c|c|c|c|c|c|c|c|c|c|c|c|}
\hline i6Py & 0,08 & $0,00^{*}$ & $-0,04^{*}$ & $0,02 *$ & $-0,08^{*}$ & $0,00^{*}$ & $-0,03^{*}$ & 0,19 & $0,01^{*}$ & 0,11 & 0,13 \\
\hline i7Py & 0,11 & $0,03^{*}$ & $0,01 *$ & $-0,01 *$ & $-0,03 *$ & $0,04^{*}$ & $0,03 *$ & $0,03^{*}$ & $-0,01 *$ & $0,00^{*}$ & $0,04 *$ \\
\hline i9Py & 0,11 & $0,03 *$ & 0,08 & 0,12 & $0,02 *$ & 0,21 & 0,13 & $0,02 *$ & $-0,10^{*}$ & $-0,08^{*}$ & $-0,08 *$ \\
\hline i10Py & 0,08 & $0,04^{*}$ & $-0,02 *$ & $0,11^{*}$ & $-0,06$ & $0,09 *$ & 0,03 & $0,07 *$ & $-0,02 *$ & $-0,04 *$ & $-0,03 *$ \\
\hline i13Рy & 0,13 & $0,03 *$ & $-0,02 *$ & $-0,02 *$ & $0,01 *$ & $-0,01 *$ & $0,01^{*}$ & 0,11 & $0,09^{*}$ & $0,07 *$ & $0,09 *$ \\
\hline i15Py & $0,02^{*}$ & $0,00^{*}$ & $-0,08^{*}$ & $0,06^{*}$ & $-0,04 *$ & $0,07 *$ & $0,00^{*}$ & $0,09^{*}$ & $0,02 *$ & $0,00^{*}$ & $-0,07 *$ \\
\hline i1 Ny & $-0,09 *$ & $0,04^{*}$ & $-0,07 *$ & $-0,19$ & $-0,06^{*}$ & $-0,09 *$ & $-0,17$ & $0,01 *$ & $0,01 *$ & $-0,02 *$ & $0,04 *$ \\
\hline i3Ny & $0,02^{*}$ & $0,10^{*}$ & $0,09^{*}$ & $0,08^{*}$ & $0,04^{*}$ & $-0,04 *$ & $-0,01^{*}$ & $-0,02^{*}$ & $-0,03^{*}$ & $-0,04 *$ & $-0,03 *$ \\
\hline i8Ny & $-0,07 *$ & $0,03^{*}$ & $0,05^{*}$ & $0,04 *$ & $-0,07^{*}$ & $-0,12$ & $-0,12$ & $0,03^{*}$ & $0,00^{*}$ & $-0,04^{*}$ & $0,00 *$ \\
\hline i14Ny & $-0,04 *$ & $0,04 *$ & $0,03 *$ & $0,04^{*}$ & $-0,08 *$ & $-0,05^{*}$ & $-0,06^{*}$ & $0,06^{*}$ & $-0,02 *$ & $-0,03 *$ & $0,01 *$ \\
\hline i4Py & 1,00 & & & & & & & & & & \\
\hline i6Py & 0,67 & 1,00 & & & & & & & & & \\
\hline i7Py & 0,63 & 0,61 & 1,00 & & & & & & & & \\
\hline i9Py & 0,41 & 0,30 & 0,36 & 1,00 & & & & & & & \\
\hline i10Py & $0,59 *$ & 0,62 & 0,58 & 0,44 & 1,00 & & & & & & \\
\hline i13Рy & 0,55 & 0,60 & 0,49 & 0,21 & 0,43 & 1,00 & & & & & \\
\hline i15Py & 0,47 & 0,35 & 0,38 & 0,32 & 0,43 & 0,39 & 1,00 & & & & \\
\hline i1Ny & $-0,35$ & $-0,26$ & $-0,29$ & $-0,20$ & $-0,31$ & $-0,33$ & $-0,41$ & 1,00 & & & \\
\hline i3Ny & $-0,30$ & $-0,34$ & $-0,26$ & $-0,18$ & 0,32 & $-0,3$ & $-0,33$ & $\mathbf{0 , 3 8}$ & 1,00 & & \\
\hline i8Ny & $-0,29$ & $-0,27$ & $-0,23$ & $-0,24$ & $-0,26$ & $-0,28$ & $-0,40$ & 0,49 & 0,79 & 1,00 & \\
\hline i14Ny & $-0,30$ & $-0,34$ & $-0,31$ & $-0,15$ & $-0,35$ & $-0,35$ & $-0,34$ & 0,48 & 0,7 & 0,72 & 1,00 \\
\hline
\end{tabular}

Nota: (x) Policlínica Piquet Carneiro Universidade do Estado do Rio de Janeiro ; ( y) Unidade de Saúde de São João de Meriti ; $\left(^{*}\right)$ nível de significância não aceitável da ordem de 5\%.

A maioria das correlações da Unidade de Saúde PPC/UERJ apresentou níveis de significância aceitáveis, próximos de zero, apenas as correlações 0,21 (i13Px e i9Px) e 0,15 (i14Nx e i9Px) afastaram-se deste nivelamento com valores de 0,0089 e 0,0724, respectivamente, muito acima dos demais. Na Unidade de Saúde de SJM foi observado correlações também não significativas, $-0,10$ para os itens i3Ny e i9Py tendo significância de 0,2202 e -0,14 para as associações dos itens i8Ny e i9Py e dos itens i14Ny x i9Py, apresentando significâncias 0,0915 e 0,0852. Ao combinar as duas Unidades de Saúde, algumas correlações mostraram-se não significativas ao associar itens, tendo sido discriminadas na Tabela 3. Das 242 correlações dos itens, 39,67\% delas não foram significativas. 
Tabela 3: Significância das correlações entre itens positivos $(\mathrm{P})$ e negativos $(\mathrm{N})$ do questionário nos ambulatórios pediátricos das Unidades de Saúde

PPC/UERJ (x) e SJM (y)

\begin{tabular}{|c|c|c|}
\hline Itens & $\begin{array}{l}\text { Correlações não } \\
\text { significantes }\end{array}$ & Níveis de significância \\
\hline i4Px & 5 & 0,$2202 ; 0,8631 ; 0,4687 ; 0,9638 ; 0,6449$ \\
\hline i6Px & 10 & $\begin{array}{c}0,6078 ; 0,5534 ; 0,7551 ; 0,9850 ; 0,8152 ; 0,9820 ; 0,5833 \\
0,7439 ; 0,3541 ; 0,9615\end{array}$ \\
\hline \multirow[t]{2}{*}{ i7Px } & 10 & 0,$7302 ; 0,2250 ; 0,7113 ; 0,9091 ; 0,8873 ; 0,6906$ \\
\hline & & 0,$5973 ; 0,6973 ; 0,9009 ; 0,9468$ \\
\hline i9Px & 11 & $\begin{array}{c}0,5834 ; 0,7439 ; 0,8246 ; 0,7638 ; 0,2257 ; 0,3287 ; 0,3040 \\
0,3185 ; 0,6542 ; 0,8347 ; 0,4538\end{array}$ \\
\hline \multirow[t]{2}{*}{ i10Px } & 10 & 0,$6995 ; 0,4004 ; 0,7685 ; 0,6237 ; 0,7341 ; 0,6848$ \\
\hline & & 0,$8165 ; 0,8366 ; 0,9188 ; 0,9359$ \\
\hline \multirow[t]{2}{*}{ i13Px } & 9 & 0,$8750 ; 0,2693 ; 0,3858 ; 0,2814 ; 0,7987 ; 0,9914$ \\
\hline & & 0,$3535 ; 0,4694 ; 0,6345$ \\
\hline \multirow[t]{2}{*}{$\mathrm{i} 1 \mathrm{Nx}$} & 9 & 0,$3866 ; 0,9818 ; 0,2447 ; 0,7901 ; 0,9740 ; 0,4009$ \\
\hline & & 0,$2916 ; 0,6323 ; 0,3919$ \\
\hline$i 3 \mathrm{Nx}$ & 11 & $\begin{array}{c}0,4643 ; 0,2615 ; 0,8597 ; 0,9084 ; 0,8372 ; 0,6329 ; 7879 ; \\
0,2354 ; 0,2811 ; 0,3119 ; 0,6428\end{array}$ \\
\hline $\mathrm{i} 8 \mathrm{Nx}$ & 10 & $\begin{array}{c}0,6032 ; 0,8658 ; 0,8213 ; 0,7420 ; 0,5806 ; 0,6824 ; 0,4185 \\
0,6663 ; 0,5245 ; 0,6304 ;\end{array}$ \\
\hline $\mathrm{i} 14 \mathrm{Nx}$ & 10 & $\begin{array}{c}0,3601 ; 0,7058 ; 0,9941 ; 0,6507 ; 0,9432 ; 0,6568 ; 0,6216 \\
0,7353 ; 0,6368 ; 0,3463\end{array}$ \\
\hline i4Py & 1 & 0,9638 \\
\hline
\end{tabular}

Esta matriz de correlação Multitraço-Multimétodo apresentou um grande número de correlações fracas entre diferentes traços (itens) medidos em diferentes locais 
(método), variando de -0,20 a 0,20, traduzindo aproximações de correlações menores que 0,30 corroborando a validade discriminante. As correlações na diagonal de validade convergente para a Unidade de Saúde PPC/UERJ variaram de -0,41 a 0,36 até 0,79, mas o limiar da validade é de 0,30 para esta validade convergente. Em SJM, as correlações variaram de -023 a 0,90, de forma análoga, deve-se observar o limiar 0,30, permitindo inferir de forma assemelhada. Estes achados permitem inferir que a validade convergente foi alcançada pelo questionário nos dois locais de aplicação.

\section{Discussão}

Na matriz Multitraço-Multimétodo, três blocos foram criados: dois triângulos multitraço-monométodo e um retângulo multitraço-multimétodo. A evidência da validade discriminante do questionário foi alcançada em função das fracas correlações entre os escores dos itens do questionário aplicados nos dois locais (SJM e PPC/UERJ). No que tange a validade convergente é importante ressaltar que nos dois triângulos multitraçomonométodo a validade convergente alcançada pode traduzir medidas do construto teoricamente semelhantes para cada dimensão em ambos locais. No entanto, a validade discriminante, também alcançada, denota a efetividade dos itens das dimensões "benefícios do acréscimo de óleos vegetais in natura na dieta infantil" e "possíveis problemas relacionados ao acréscimo de óleos vegetais in natura na dieta infantil" para discriminar o comportamento alimentar dos responsáveis pela alimentação da criança tendo em vista sua cognição sobre alimentação para aderir ou não a tal prática dietética.

Deon et al. [10] ao avaliar a adaptação transcultural brasileira do Atopic Dermatitis Module - DISABKIDAS, módulo fibrose cística de crianças e adolescentes encontraram correlações que variaram de 0,14 a 0,61 , sendo que na maioria das vezes, maiores que 0,40 para a validade convergente. No presente estudo, há semelhança com os achados dos autores em relação às correlações nas diagonais de validade convergentes tanto para PPC/UERJ e SJM.

Das 242 correlações dos itens, 39,67\% delas não foram significativas, explicado pelas diferentes gestões inerentes a cada Unidade de Saúde, pois na PPC/UERJ há uma Divisão de Nutrição, na qual os nutricionistas seguem as recomendações do Ministério da Saúde, enquanto esta orientação não se verifica em SJM. No que tange a validade discriminante, ambos os estudos alcançaram resultados aceitáveis, logo estes achados 
indicam comportamentos diferenciados entre os respondentes dos dois locais tendo em vista suas peculiaridades.

Brown [11] afirma que um dos usos mais elegantes de análise fatorial confirmatória em validação de construto é a análise de Multitraço-Multimétodo por matrizes, onde as validades, convergente e discriminante, das dimensões são avaliadas no contexto, excluindo a influência de diferentes métodos de avaliação.

A contribuição do estudo foi utilizar um algoritmo que trata da análise inferencial, tomando-se como entrada do sistema a matriz de correlação sem negligenciar a fidedignidade dos seus valores, e que adaptou-se ao desenvolvimento de escalas de comportamento alimentar.

O instrumento pode ser utilizado em ambulatórios para avaliar o conhecimento dos responsáveis pela alimentação da criança e orientá-los segundo sua cognição em relação ao uso do óleo vegetal in natura de acréscimo na dieta infantil.

\section{Conclusão}

Conclui-se que o instrumento proposto apresentou evidências de validade discriminante e convergente. É importante destacar que o estudo mostrou que os responsáveis pela alimentação da criança possuem cognições diferenciadas sobre a prática do uso do óleo vegetal in natura de acréscimo na dieta infantil, sendo necessário considerar o tipo de gestão de cada Unidade de Saúde na área de Nutrição no que se refere a protocolos de atendimento nutricional.

\section{Referências}

[1] BRASIL. Ministério da Saúde (MS): Política Nacional de Atenção Integral à Saúde da Criança: orientações para implementação. Brasília: Ministério da Saúde, 2018.

[2] BRASIL. Ministério da Saúde (MS): Dez passos para uma alimentação saudável. Guia alimentar para crianças menores de dois anos: um guia para o profissional da saúde na atenção básica. Brasília: Ministério da Saúde, 2013.

[3] BROWN T.A.: Confirmatory factor analysis for applied research. New York: The Guilford Press, 2006.

[4] CAMPBELL D.T, FISKE D.W.: Convergent and discriminant validation by the multitrait-multimethod matrix. Psychological Bulletin, v.56(2), p.81-105,1959. 
[5] DEON K.C. et al.: Análise psicométrica inicial da versão brasi1eira do DISABKIDS Atopic Dermatitis Module. Revista de Saúde Pública, v.45(6), p.1072-1078, 2011.

[6] JORGE N., LOPES M.R.V.: Determinação de compostos polares totais em óleos e gorduras de frituras. Higiene Alimentar, v.19(134), p.46-50, 2005.

[7] KOLETZKO, B.: Ácidos graxos poliinsaturados de cadeia longa (LCPUFA) na dieta de lactentes prematuros. In: Seminário Nestlé Nutrition. Vevey, Suíça: Nutrition Workshop Series, 1998.

[8] LANZILLOTTI H.S. et al.: Estrutura fatorial do questionário de adoção do óleo vegetal in natura de acréscimo na dieta infantil. Ciência \& Saúde Coletiva, v.24(2), p.465474, 2019. Disponível em: https://doi.org/10.1590/1413-81232018242.29612016

[9] MARTIN C.A et al.: Ácidos graxos poliinsaturados ômega-3 e ômega-6: importância e ocorrência em alimentos. Revista Nutrição, v.19(6), p.761-770, 2006.

[10] NÚCLEO DE ESTUDOS E PESQUISAS EM ALIMENTAÇÃO (NEPA): Tabela Brasileira de composição de alimentos. 2 ed. Campinas, São Paulo: NEPA-UNICAMP, 2006.

[11] SANT'ANA L.S.: Biodisponibilidade dos lipídeos. In: Cozzolino S.M.F. Biodisponibilidade de nutrientes. Barueri: Manole, 2005. 